\title{
Solitary waves on a ferrofluid jet
}

\author{
M. G. BLYTH AND E. I. P $\breve{A} R \breve{A} U$ \\ School of Mathematics, University of East Anglia, Norwich, NR4 7TJ, England, UK
}

(Received 14 May 2014)

The propagation of axisymmetric solitary waves on the surface of an otherwise cylindrical ferrofluid jet subjected to a magnetic field is investigated. An azimuthal magnetic field is generated by an electric current flowing along a stationary metal rod which is mounted along the axis of the moving jet. A numerical method is used to compute fully-nonlinear travelling solitary waves and predictions of elevation waves and depression waves by Rannacher \& Engel (2006) using a weakly-nonlinear theory are confirmed in the appropriate ranges of the magnetic Bond number. New nonlinear branches of solitary wave solutions are identified. As the Bond number is varied, the solitary wave profiles may approach a limiting configuration with a trapped toroidal-shaped bubble, or they may approach a static wave (i.e. one with zero phase speed). For a sufficiently large axial rod, the limiting profile may exhibit a cusp.

\section{Introduction}

Since their first observation by Scott Russell on the Edinburgh-Glasgow Union canal in 1834, solitary waves have been the subject of intense scientific interest. Hydrodynamic solitary waves are localised structures which propagate along the surface of a fluid at constant speed while preserving their form. To date most theoretical studies have been devoted to the subject of two-dimensional solitary waves (see e.g. Miles (1980) for a review), but more recently computations of three-dimensional solitary waves have been reported (Părău et al. 2005). Mathematically, solitary wave solutions emerge as bifurcations from the neutral wave branch of non-amplifying and non-decaying travelling waves in an inviscid fluid, either from the long-wave limit or from the minimum point in the corresponding dispersion curve relating wave speed to wave number (e.g. Dias \& Kharif 1999).

In this paper we examine the propagation of axisymmetric solitary waves along the surface of an inviscid liquid jet in the absence of gravity. It is well known that such a jet is linearly unstable to long-wave disturbances with wavelength greater than the circumference of the jet (Rayleigh 1878). Periodic disturbances of wavelength less than this are stable and propagate with fixed amplitude (Vanden-Broeck et al. 1998; Osborne \& Forbes 2001). Since the long-wave limit is unstable, and the dispersion curve for the fixed-amplitude travelling waves covers all possible speeds and does not have a minimum, there are not expected to be any bifurcations to solitary wave solution branches, nor have any such bifurcations been found.

It has been observed that a magnetic field can be used to suppress the long-wave Rayleigh instability and stabilise a jet composed of a ferrofluid (see Arkhipenko et al. (1980); Rosensweig (1985) for a review). Ferrofluids are liquids which contain in suspension tiny magnetizable particles; consequently, the fluid experiences a body force in the presence of an external magnetic field. Working on the basis that a jet can be 
stabilised in this way, Bashtovoi et al. (1983) showed that the classical KdV equation provides a model for describing weakly-nonlinear disturbances on the surface of the jet. Taking a set-up in which a ferrofluid jet is subjected to an azimuthal magnetic field generated by a thin, current-carrying wire positioned along the axis of the jet, Rannacher \& Engel (2006), henceforth referred to as RE, confirmed via a linear stability analysis that the jet is indeed stabilised and derived the KdV equation describing axisymmetric weakly-nonlinear disturbances. They identified solitary wave solutions to this equation and, specifically, showed that these are elevation waves (with a hump at the centre of the wave) if $1<B<3 / 2$, they are depression waves (with a dip at the centre of the wave) if $3 / 2<B<9$, and they are elevation waves if $B>9$, where $B$ is the magnetic Bond number. They also studied interactions between two solitary waves. Note that when $B<1$ the jet is unstable (Arkhipenko \& Barkov 1980).

In this paper we revisit the problem studied by RE with the primary goal of extending the previous results into the fully-nonlinear regime. We consider a more general set-up in which the magnetic field is generated by a current-carrying rod of arbitrary radius. One advantage of this is that it allows us to compare results with the experimental observations which have recently been reported by Bourdin et al. 2010. The structure of the remainder of the paper is as follows. In section 2 we formulate the problem. In section 3 we conduct a linear stability analysis for axisymmetric disturbances. In section 4 we discuss static solutions for which there is no flow. In section 5 we carry out a far-field analysis. In sections 6 and 7 we describe our numerical method for the fully-nonlinear computations and present our results. Finally, in section 8 we discuss our findings.

\section{Problem formulation}

We consider the inviscid, incompressible, irrotational flow of a liquid jet of density $\rho$ flowing along the outside of a solid cylindrical metal rod of radius $b^{*}$. The liquid flows in the $x^{*}$ direction of a cylindrical polar coordinate system $\left(r^{*}, \theta, x^{*}\right)$. In the basic configuration the jet surface is a circular cylinder with radius $a^{*}$. Henceforth we assume conditions of asixymmetry so that all variables are independent of $\theta$. The liquid in the jet is assumed to be a ferrofluid, that is to say a liquid that can be magnetized and thereby experience a body force in the presence of an external magnetic field. The axial rod carries a current $I$ in the $x^{*}$ direction which generates an azimuthal magnetic field $\mathbf{B}=\mu_{0} I \mathbf{e}_{\theta} /\left(2 \pi r^{*}\right)$, where $\mathbf{e}_{\theta}$ is the unit vector in the $\theta$ direction. The magnetic field induces a radial body force in the ferrofluid given by (e.g. Rosensweig 1985)

$$
\mathbf{F}=\left(\chi / \mu_{0}\right) \mathbf{B} \cdot \nabla \mathbf{B}=-\frac{\mu_{0} \chi I^{2}}{4 \pi^{2} r^{* 3}} \mathbf{e}_{r},
$$

where $\mathbf{e}_{r}$ is the unit vector in the $r^{*}$ direction, and $\chi$ is the magnetic susceptibility of the ferrofluid and $\mu_{0}=4 \pi \times 10^{-7} \mathrm{Hm}^{-1}$ is the magnetic permeability in a vacuum.

It is convenient to introduce the dimensionless spatial variables $(r, x)=\left(r^{*}, x^{*}\right) / a^{*}$ and the dimensionless time $t=t^{*} /\left(a^{* 3} \rho / \gamma\right)^{1 / 2}$, where $\gamma$ is the surface tension at the free surface of the jet. The dimensionless rod radius is defined to be

$$
b=\frac{b^{*}}{a^{*}} .
$$

Assuming the presence of waves on the jet surface, we work in a frame of reference travelling at the as yet unknown dimensionless wave-speed $c$, and introduce the travellingwave coordinate $z=x-c t$. In the travelling frame the surface of the jet is located at 
$r=S(z)$. The flow in the fluid is described by the Laplace equation

$$
\nabla^{2} \phi=0
$$

where $\phi$ is the velocity potential. At the axial rod we demand that

$$
\phi_{r}=0
$$

on $r=b$. At the free surface, the kinematic condition requires that

$$
\phi_{r}=\phi_{z} S_{z}
$$

on $r=S$. Applying the Bernoulli equation at the free surface, and making use of the kinematic condition (2.5), we find that

$$
\frac{1}{2} \phi_{z}^{2}\left(1+S_{z}^{2}\right)-\frac{S_{z z}}{\left(1+S_{z}^{2}\right)^{3 / 2}}+\frac{1}{S\left(1+S_{z}^{2}\right)^{1 / 2}}-\frac{B}{2 S^{2}}=\frac{1}{2} c^{2}+1-\frac{B}{2},
$$

where the first term is evaluated at $r=S$ and we have used the condition that $\phi_{z} \rightarrow-c$ as $|z| \rightarrow \infty$. The magnetic Bond number is given by

$$
B=\frac{\mu_{0} \chi I^{2}}{4 \pi^{2} \gamma a^{*}}
$$

The second and third terms on the left hand side of (2.6) represent the capillary pressure at the surface of the jet, and the fourth term on the left hand side represents the effect of the magnetic stress.

The Bernoulli constant on the right hand side has been fixed by demanding that $S \rightarrow 1$ as $|z| \rightarrow \infty$. Curiously, the same Bernoulli constant is obtained by taking the alternative limit $S \rightarrow \mathcal{S}_{0}$, where $\mathcal{S}_{0}=B /(2-B)$, as $|z| \rightarrow \infty$. At first glance, it appears then that a second equilibrium state is possible in the far-field, counter to physical intuition. To reconcile the issue, we note that under the transformation

$$
\phi=\sqrt{\mathcal{S}_{0}} \hat{\phi}, \quad c=\frac{1}{\sqrt{\mathcal{S}_{0}}} \hat{c}, \quad S=\mathcal{S}_{0} \hat{S}, \quad(z, r)=\mathcal{S}_{0}(\hat{z}, \hat{r}), \quad B=2-\hat{B},
$$

the problem (2.3)-(2.6) is unchanged and, consequently, solutions computed by taking $S=\mathcal{S}_{0}$ in the far-field are simply transformations of those obtained by taking $S=1$ in the far-field. Therefore, as is expected on physical grounds, it is sufficient to consider solutions for which $S \rightarrow 1$ as $|z| \rightarrow \infty$.

\section{Small amplitude theory}

Under a small perturbation, the jet surface is displaced to the new location,

$$
S=1+\delta A \mathrm{e}^{\mathrm{i} k z},
$$

where $\delta \ll 1$ is a small parameter, $A$ is a constant complex amplitude to be determined, and $c$ and $k$ are the complex wave speed and wavenumber of the disturbance respectively. The velocity potential is perturbed similarly, so that

$$
\phi=-c z+\delta f(r) \mathrm{e}^{\mathrm{i} k z},
$$

where $f(r)$ is to be found, and the first term on the right hand side corresponds to the uniform velocity of the undisturbed jet. Substituting (3.1) and (3.2) into the problem given by (2.3)-(2.6), we ultimately derive the dispersion relation (Arkhipenko \& Barkov 
1980)

$$
c^{2}=\frac{1}{k}\left(\frac{I_{1}(k) K_{1}(k b)-I_{1}(k b) K_{1}(k)}{I_{1}(k b) K_{0}(k)+I_{0}(k) K_{1}(k b)}\right)\left(k^{2}-1+B\right),
$$

where $I_{0}, I_{1}$ and $K_{0}, K_{1}$ are modified Bessel functions. Note that the fraction inside the first bracket in (3.3) is positive for all positive $k$ (Radwam 1988). Formula (3.3) reduces to that given by RE in the limit $b \rightarrow 0$. In the long-wave limit $k \rightarrow 0$ we find that

$$
c^{2}=c_{0}^{2}+O\left(k^{2}\right), \quad c_{0}=\left(\frac{B-1}{2}\right)^{1 / 2}\left(1-b^{2}\right)^{1 / 2} .
$$

A key observation to be made from (3.3) is that the jet is unstable when $B<1$ in which case there is a range of wavenumbers $k<(1-B)^{1 / 2}$ for which $c$ is purely imaginary and disturbances are amplified. When $B \geq 1$, the wave speed $c$ is real and neutral waves exist for arbitrary wave number $k$. It follows that a magnetic field of sufficient intensity can stabilise the Rayleigh capillary mode responsible for jet break-up under normal conditions. This result underpins the studies of RE and Bourdin et al. (2010) as it provides a firm basis for both the theoretical and experimental search for solitary wave solutions, which appear as bifurcations from the neutral wave branch. A second important observation from (3.3) is that a minimum, $c_{M}$, appears in the dispersion curve when $B>B_{2}$, where $B_{2}$ depends on the radius of the axial rod $b$. For small $b$, we find

$$
B_{2}=9+24 b^{2}+O\left(b^{4}\right) .
$$

The general dependence of $B_{2}$ on $b$ is readily found but the formula is algebraically complicated and is not given here. Drawing an analogy with capillary-gravity waves on water of finite depth (see the discussion in the next paragraph), we expect that when $B$ is below the threshold for a minimum, a branch of solitary wave solutions should appear as a bifurcation from the neutral-wave branch at $k=0$. When $B$ exceeds the threshold and a minimum is present, then the solitary wave branch should bifurcate from the neutral branch at $k=k_{M}$, where $c\left(k_{M}\right)=c_{M}$.

Figure 1( $a$ ) shows typical dispersion curves for $b=0$ (thin axial rod) corresponding to (3.3) for a number of different values of $B$. Figure 1(b), also for the thin-rod $b=$ 0 case, shows the locus of the minimum $c_{M}$ versus Bond number $B$ for $B \geq 9$ and also $c_{0}$ versus $B$. The shaded areas indicate regions in which weakly-nonlinear theory is expected to hold. Since the full weakly-nonlinear theory for the present problem has not yet been developed, it is useful to draw a parallel with gravity-capillary waves in twodimensional free-surface flow, on which topic there has been a great deal of discussion in the literature (Dias \& Kharif 1999, Vanden-Broeck 2010). For such waves, the dispersion curves have the same qualitative characteristics as here, with a minimum appearing when the dimensionless surface tension parameter is below a threshold. Above this threshold the standard KdV equation provides an accurate description of the weakly-nonlinear dynamics; around the threshold, the standard KdV equation breaks down but a fifth order modified KdV equation may be derived for weakly-nonlinear predictions (Hunter \& Vanden-Broeck 1983). Below the threshold, a weakly-nonlinear description is sought around the wave speed at the minimum of the dispersion curve. Such an analysis leads to the nonlinear Schrödinger equation (Akylas 1993). For the present problem, around the region I indicated in figure $1(b)$, the KdV equation is expected to hold, and indeed this equation has already been derived in the thin-rod case $b=0$ by Bashtovoi \& Foigel (1983) (see also RE). This equation breaks down at $B=9$. Above this limit, in region 
(a)

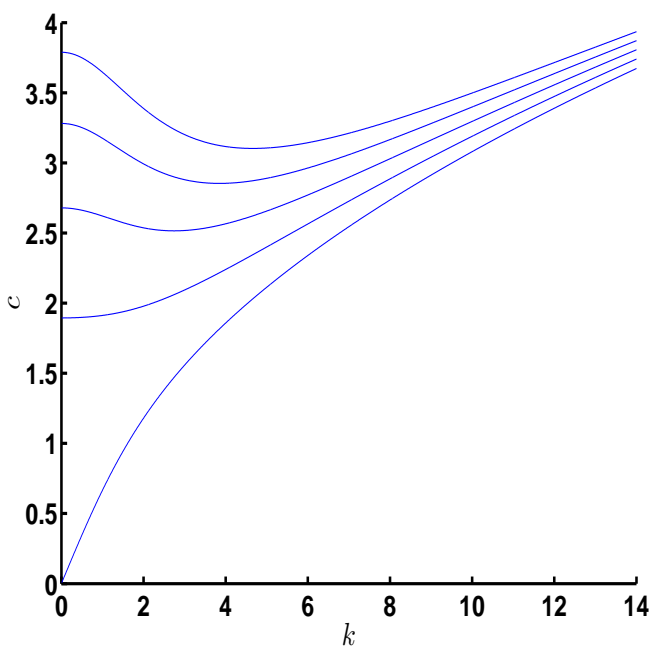

$(b)$

Figure 1. (a) Dispersion curves according to 3.3 for $b=0$ and for $B=1$ to $B=30$ in equally-spaced intervals from the lower curve to the upper curve. The curves meet the vertical axis at $c=c_{0}$ and for $B>9$ there is a minimum at $c=c_{M}$. (b) Sketch of the domains of interest for $b=0$ (no axial rod). The broken line shows $c_{0}=(B-1)^{1 / 2} / \sqrt{2}$ versus Bond number $B$, and the solid line shows $c_{M}$ versus $B$. The two dotted lines indicate the point $c_{M}=2, B=9$ at which the minimum emerges.

II marked in figure 1(b), it should be possible to derive an analogue of the nonlinear Schrödinger equation.

The focus of the present study is on the numerical computation of fully-nonlinear solitary waves, and for this reason we do not attempt a weakly-nonlinear analysis. The discussion is helpful, however, as it serves as a guide to the type of waves, and the number of solution branches, which we should expect over different ranges of the Bond number. The KdV equation derived by Bashtovoi \& Foigel (1983) (see also RE) for the case $b=0$ suggests that, for general $b$, there are small-amplitude elevation solitary waves with $c<c_{0}$ in the range $1 \leq B \leq B_{1}$ and small-amplitude depression solitary waves with $c<c_{0}$ in the range $B_{1} \leq B \leq B_{2}$. For $B>B_{2}$ the nonlinear Schrödinger equation provides a clue to the possible solution branches, as will be discussed below. We note that the dependence of $B_{1}$ on $b$ is at present unclear as it should be determined through a weakly nonlinear analysis similar to that performed by RE. Here we note simply that RE found that $B_{1}=1.5$ when $b=0$. Further insight into the possible solution branches is obtained by considering the case when there is no motion in the liquid and seeking stationary solitary wave solutions supported by a balance between surface tension and the magnetic body force at the free surface. This is discussed next.

\section{Static solutions}

Here we consider the possible equilibrium configurations of the liquid surface in the absence of any flow. For a quiescent fluid, the Bernoulli equation (2.6) reduces to

$$
-\frac{S_{x x}}{\left(1+S_{x}^{2}\right)^{3 / 2}}+\frac{1}{S\left(1+S_{x}^{2}\right)^{1 / 2}}-\frac{B}{2 S^{2}}=1-\frac{B}{2} .
$$


To proceed it is helpful to recast this equation in the form of the two-dimensional conservative dynamical system

$$
\frac{\mathrm{d}}{\mathrm{d} x}\left(\begin{array}{l}
S \\
\alpha
\end{array}\right)=\left(\begin{array}{c}
\sin \alpha \\
B / 2-1+\cos \alpha / S-B /\left(2 S^{2}\right)
\end{array}\right),
$$

where $\alpha$ is the angle between the tangent to the curve and the horizontal. Fixed points of this system occur at

$$
(S, \alpha)=(1,2 n \pi), \quad\left(\mathcal{S}_{0}, 2 n \pi\right), \quad\left(-\mathcal{S}_{0},(2 n+1) \pi\right), \quad(-1,(2 n+1) \pi),
$$

where $\mathcal{S}_{0}=B /(2-B)$ and $n$ is an integer. The last fixed point with $S=-1$ is removed from further consideration on physical grounds.

The energy, $E$, associated with the system (4.2) is given by

$$
E=S \cos \alpha+\frac{1}{2}\left(\frac{B}{2}-1\right) S^{2}-\left(\frac{B}{2}\right) \log S,
$$

and trajectories in the $(S, \alpha)$ plane are given by lines of constant $E$. The qualitative structure of the phase portrait in this plane depends on the size of the Bond number. As has already been discussed, solutions for $0<B<1$ may be obtained by a simple transformation from those for $B>1$ and so we may confine our attention to the latter range. For $B>1$ the fixed points $(S, \alpha)=(1,2 n \pi)$ correspond to a cylindrical interface, and a standard local analysis reveals that they are saddle points. For $1<B<2$, we have $\mathcal{S}_{0}>0$ and of those listed in (4.3) the fixed point at $\left(\mathcal{S}_{0}, 0\right)$ is the only other point of physical relevance. It can be readily shown that since $E$ attains its minimum value here, this point is a centre (e.g. Strogatz, p. 163). A typical portrait in this Bond number range is shown in figure $2(a)$ for the case $B=1.25$. The homoclinic orbit which connects the point $(S, \alpha)=(1,0)$ to itself corresponds to a deformed free surface shape with a single hump, as is illustrated in figure $2(b)$. This solution was computed numerically using Runge-Kutta integration. The closed orbits contained within the homoclinic orbit in figure $2(a)$ correspond to static solutions with a periodic wavy profile. The heteroclinic orbit connecting the neighbouring saddle points at $\alpha=2 n \pi$ and $\alpha=2(n+1) \pi$ corresponds to a physical profile with a point of self-intersection.

If $B>2$, then $\mathcal{S}_{0}<0$ and the physically relevant fixed points are the saddles at $(S, \alpha)=(1,2 n \pi)$ and the fixed points at $\left(-\mathcal{S}_{0},(2 n+1) \pi\right)$, which can be shown to be centres. A typical phase portrait in this Bond number range is shown in 2(a) for the case $B=30$. Two heteroclinic orbits connect each neighbouring pair of saddle points at $\alpha=2 n \pi$ and $\alpha=2(n+1) \pi$. The free surface depression profile $S$ corresponding to the lower heteroclinic orbit is shown in figure $2(b)$ and evidently self-intersects so that it appears not to be a physically acceptable solution. The elevation profile corresponding to the upper heteroclinic orbit also self-intersects but it is not shown here. When $B=$ 2 the only fixed points are the saddle points at $(S, \alpha)=(1,2 n \pi)$. Heteroclinic orbits corresponding to depression waves connect neighbouring saddle points in the phase plane, and the corresponding physical profiles self-intersect as in the lower panel of figure 2(b).

Although the free-surface depression profiles corresponding to the heteroclinic orbits connecting neighbouring saddles self-intersect, they are still open to physical interpretation. Hunter \& Vanden-Broeck (1983) constructed limiting stationary solutions for two-dimensional gravity-capillary solitary waves in finite depth for which the wave profile just touches the bottom in a cusp. Analogous solutions may be constructed here. The heavy line overlaid onto the lower self-intersecting profile in figure $2(b)$ illustrates one half of such a symmetric cusp solution which just touches the axial rod (whose location is illustrated with a broken line). The other half of the solution may be constructed by 
(a)
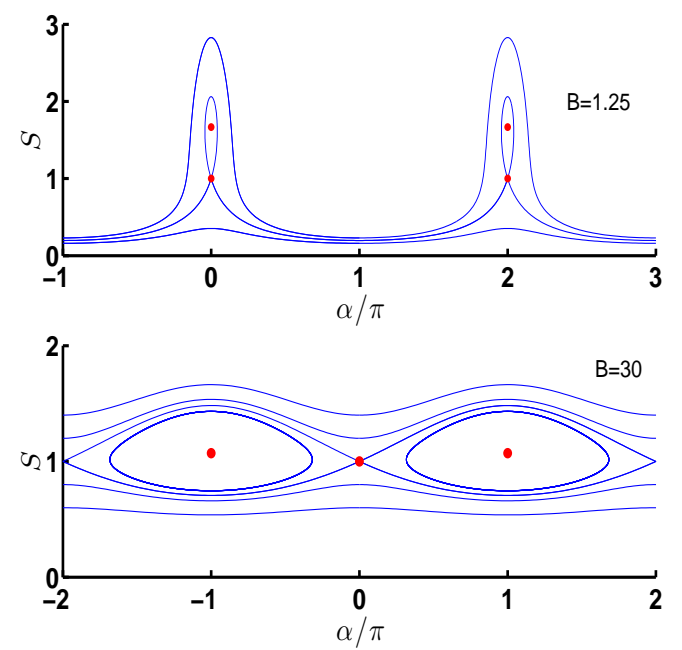

(b)
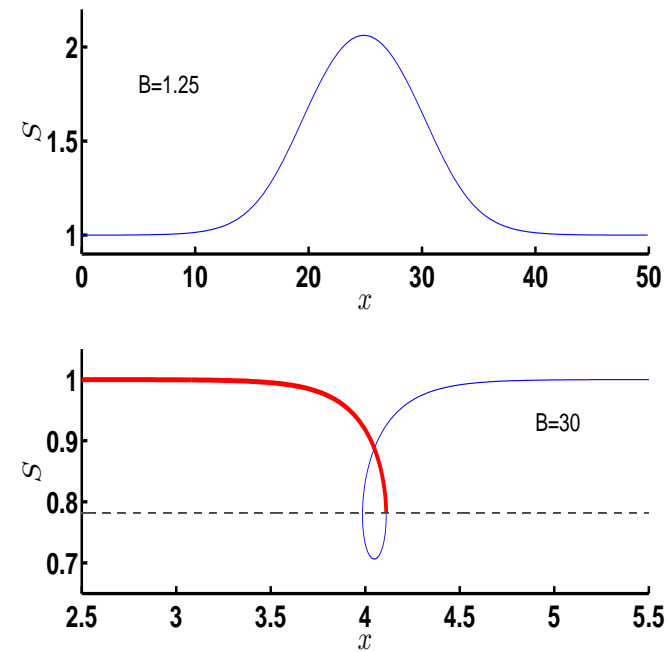

Figure 2. (a) Phase portraits for the system (4.2) at $B=1.25$ and $B=30$. Fixed points are indicated by the solid dots. (b) The static solitary wave profiles correspond to the homoclinic/heteroclinic orbits in the phase portraits in $(a)$. The heavy line overlaid on the lower figure shows one half of a symmetric cusp solution.

reflection in the obvious way. The heavy line as shown meets the axial rod at a contact angle of $\pi / 2$. Cusp solutions with contact angle smaller than $\pi / 2$ may be extracted for any axial rod radius chosen between the broken line and the surface level $S=1$.

To determine the permissible range of cusp solutions, we restrict attention to the range $0 \leq \alpha<2 \pi$ without loss of generality. The energy level on the pertinent heteroclinic orbit is $E=(B+2) / 4$. The physical profile corresponding to this orbit meets the rod when at $\alpha=\pi / 2$ and $S=S_{m}$, say, in a cusp of maximum permissible contact angle $\pi / 2$. Then from (4.4) we have that

$$
\frac{1}{2}\left(\frac{B}{2}-1\right) S_{m}^{2}-\left(\frac{B}{2}\right) \log S_{m}=\frac{B}{4}+\frac{1}{2} .
$$

As discussed above, we are concerned only with the range $B>1$. In this range we find that $S_{m}$ is a monotonic increasing function of $B$, and the minimum value at $B=1$ is given by $S_{m}=0.218$. Also $S_{m} \rightarrow 1^{-}$as $B \rightarrow \infty$.

To summarise the results of this section, we have found that in the range $1 \leq B<2$ there exist smooth, static, pulse-like free surface profiles. In the range $B>1$ solutions featuring a cusp exist and may be interpreted physically provided the cusp occurs at the surface of the axial rod; this is possible if $b>S_{m}(B)$. In the range $B>2$ no smooth solutions exist.

\section{Far-field analysis}

Returning to the wave-propagation problem, in this section we examine the rate and manner of the decay back to the uniform state as $z \rightarrow \infty$. A long way downstream we seek a solution which is a small perturbation about the uniform state by writing

$$
S=1+A \mathrm{e}^{-\mu z}
$$


for constant $A$ to be found, and investigate possible values of the constant $\mu$, which is in general complex. Similarly we expand the velocity potential by writing

$$
\phi=-c z+\mathrm{e}^{-\mu z} f(r),
$$

where $f$ is a function to be determined. Demanding that $\phi$ satisfies Laplace's equation (2.3), we find that $f(r)=a_{1} J_{0}(\mu r)+a_{2} Y_{0}(\mu r)$, where $a_{1}$ and $a_{2}$ are constants and $J_{0}$ and $Y_{0}$ are Bessel functions. To satisfy the boundary conditions we require,

$$
\left(\begin{array}{ccc}
c \mu J_{0}(\mu) & c \mu Y_{0}(\mu) & \mu^{2}+(1-B) \\
J_{1}(\mu) & Y_{1}(\mu) & -c \\
J_{1}(\mu b) & Y_{1}(\mu b) & 0
\end{array}\right)\left(\begin{array}{l}
\alpha \\
\beta \\
A
\end{array}\right)=0
$$

where $J_{1}$ and $Y_{1}$ are Bessel functions. The rows of this matrix enforce the Bernoulli condition at the free surface (2.6), the kinematic condition at the free surface (2.5), and the no-penetration condition at the axial rod (2.4), respectively. Demanding a non-trivial solution we set the determinant of the matrix on the left hand side of (5.3) to zero to yield a nonlinear equation for the decay constant $\mu$.

When $c=0$ and there is no flow, the appropriate decay rate is given by

$$
\mu=(B-1)^{1 / 2} \text {. }
$$

Note that the other possible values of $\mu$ arising at $c=0$, namely the zeros of $\mathcal{Q} \equiv$ $J_{1}(\mu) Y_{1}(\mu b)-J_{1}(\mu b) Y_{1}(\mu)=0$, must be disregarded since they correspond to the degenerate case $A=0$. They represent exact separable solutions of Laplace's equation inside the annular region $b \leq r \leq 1$, which satisfy the boundary conditions at $r=b$ and $r=1$, and which decay at either $z=\infty$ or at $z=-\infty$ but not both. The corresponding values of $\mu$ are therefore inadmissible as they do not describe the far-field decay of globally acceptable solutions.

In figure 3( $a$ ) we show the real and imaginary parts of the decay rate $\mu$ for the case $b=0.1$ and $B=1.25$. The decay rate vanishes at $c=c_{0}=0.35$. For $c<c_{0}, \mu$ is real and so solitary wave solutions are expected to decay monotonically in the far field. For $c>c_{0}$, the decay rate $\mu$ is purely imaginary, suggesting that only periodic wave solutions are possible in this range, in agreement with the linear theory presented in section 3 . Figure 3) (b) shows the more complicated picture which emerges for the decay rates for the larger Bond number $B=30$. The dot-dashed lines in the figure indicate decay rate branches which we believe to be spurious since they connect to the degenerate case with $A=0$ at $c=0$ described above. The curves are shown here for completeness: they illustrate the need to be careful when picking out the decay rate as it does not necessarily correspond to the smallest (in real part) computed value of $\mu$. Immediately below the minimum in the dispersion curve (see the uppermost curve in figure 1 $a$ ), that is for $c<c_{M}=3.1$, the decay rate is complex, suggesting that waves have oscillatory decay in the far-field. (This is what happens for two-dimensional gravity-capillary waves.) Somewhat unexpectedly, however, the decay rate becomes real when $c<1.1$, suggesting monotonic decay in the far-field. We will pick up on this point again in section 7 In the range $c_{M}<c<c_{0}$, there are two decay rates both of which are purely imaginary, suggesting that only waves with non-decaying periodic tails are permissible.

We have examined the decay of the static solutions in section 4 and confirmed excellent agreement with the decay rates predicted here at $c=0$ over a range of different Bond numbers. For $c>0$ the decay rates give an indication of the kind of behaviour to be expected in the far-field and, moreover, provide a quantitative means of corroborating the full numerical solutions to be discussed below. 
$(a)$

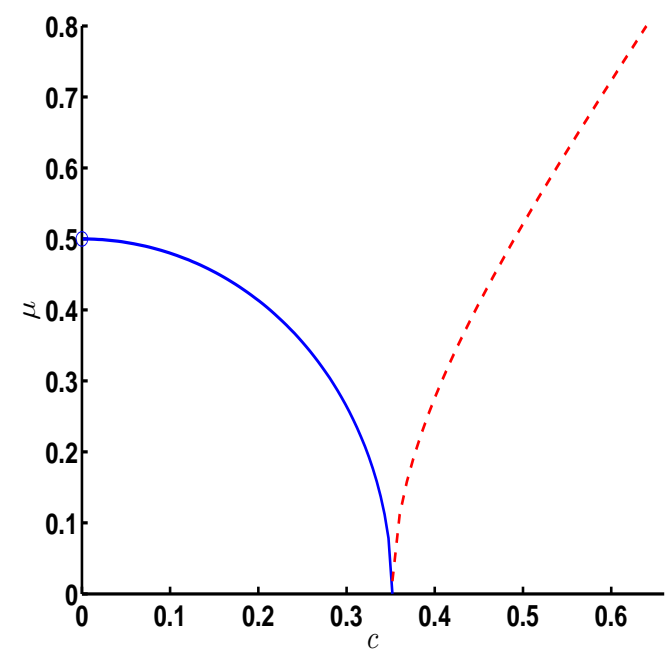

(b)

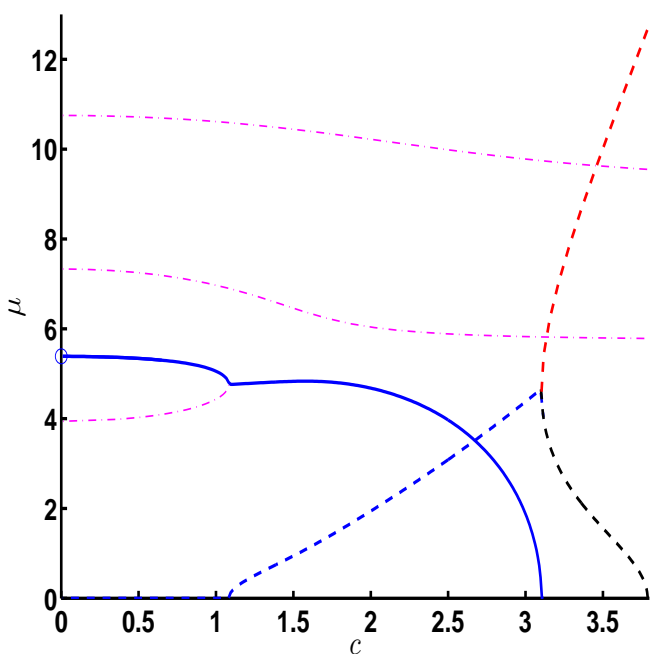

Figure 3. Complex decay rate against wave speed $c$ for $b=0.1$ and $(a) B=1.25$ and $(b)$ $B=30$. The real/imaginary parts of $\mu$ are shown with solid/broken lines. In $(a) c_{0}=0.35$, and there is one real rate in $0<c<c_{0}$ and one imaginary rate in $c>c_{0}$ within the range shown. In $(b) c_{0}=3.8$ and $c_{M}=3.1$. The decay rate is complex in the range $1.1<c<c_{M}$ and there are two imaginary rates in $c_{M}<c<c_{0}$. The dot-dashed lines in (b) correspond to spurious $\mu$ values which connect to zeros of $\mathcal{Q}$ at $c=0$. In both $(a)$ and $(b)$ the circles at $c=0$ indicate the rates $\mu=(B-1)^{1 / 2}$.

\section{Numerical method}

To compute fully-nonlinear solitary wave solutions, we use a finite-difference method based on a formulation originally due to Jeppson (1970). The same approach has been used successfully by Vanden-Broeck et al. (1998) to compute periodic axisymmetric capillary waves.

First we introduce the Stokes streamfunction $\psi$ defined so that

$$
u=-\frac{1}{r} \frac{\partial \psi}{\partial z}=\frac{\partial \phi}{\partial r}, \quad w=\frac{1}{r} \frac{\partial \psi}{\partial r}=\frac{\partial \phi}{\partial z},
$$

where $u$ and $w$ are the velocity components in the $r$ and $z$ directions respectively. Following Jeppson (1970), we have that

$$
\frac{\partial r}{\partial \phi}=-\frac{1}{J} \frac{\partial \psi}{\partial z}, \quad \frac{\partial r}{\partial \psi}=\frac{1}{J} \frac{\partial \phi}{\partial z}, \quad \frac{\partial z}{\partial \phi}=\frac{1}{J} \frac{\partial \psi}{\partial r}, \quad \frac{\partial z}{\partial \psi}=-\frac{1}{J} \frac{\partial \phi}{\partial r},
$$

where

$$
J=\frac{\partial \phi}{\partial z} \frac{\partial \psi}{\partial r}-\frac{\partial \phi}{\partial r} \frac{\partial \psi}{\partial z}, \quad J=r\left[r^{2}\left(\frac{\partial r}{\partial \psi}\right)^{2}+\left(\frac{\partial r}{\partial \phi}\right)^{2}\right]^{-1}
$$

We note that to obtain the latter of (6.3) we have used the relations

$$
\frac{\partial z}{\partial \psi}=-\frac{1}{r} \frac{\partial r}{\partial \phi}, \quad \frac{\partial r}{\partial \psi}=\frac{1}{r} \frac{\partial z}{\partial \phi},
$$

which are themselves found by substituting (6.2) into (6.1).

The idea is to reformulate the problem (2.3) -(2.6) in the $(\phi, \psi)$ plane and to solve for the unknowns $r(\phi, \psi)$ and $z(\phi, \psi)$. The physical domain of interest in the $(r, z)$ plane 
corresponds to the rectangular region

$$
-\infty<\phi<\infty, \quad 0 \leq \psi \leq \psi_{S}
$$

in the $(\phi, \psi)$ plane, where $\psi_{S}=c\left(1-b^{2}\right) / 2$. The axial rod is located at $\psi=0$ and the free surface of the jet is located at $\psi=\psi_{S}$. We recast Laplace's equation (2.3) in the form

$$
r^{3} \frac{\partial^{2} r}{\partial \psi^{2}}+r \frac{\partial^{2} r}{\partial \phi^{2}}+r^{2}\left(\frac{\partial r}{\partial \psi}\right)^{2}-\left(\frac{\partial r}{\partial \phi}\right)^{2}=0 .
$$

The no-normal-flow condition at the axial rod (2.4) becomes

$$
\frac{\partial r}{\partial \phi}=0
$$

at $\psi=0$. The Bernoulli condition (2.6) becomes

$$
\frac{1}{2}\left[r^{2}\left(\frac{\partial r}{\partial \psi}\right)^{2}+\left(\frac{\partial r}{\partial \phi}\right)^{2}\right]^{-1}-\kappa-\frac{B}{2 r^{2}}=\frac{1}{2} c^{2}+1-\frac{B}{2}
$$

at $\psi=\psi_{S}$, where

$$
\begin{aligned}
\kappa=\left(r \frac{\partial r}{\partial \psi} \frac{\partial^{2} r}{\partial \phi^{2}}-\left(\frac{\partial r}{\partial \phi}\right)^{2} \frac{\partial r}{\partial \psi}-r \frac{\partial r}{\partial \phi}\right. & \left.\frac{\partial^{2} r}{\partial \phi \partial \psi}\right)\left[\left(\frac{\partial r}{\partial \phi}\right)^{2}+r^{2}\left(\frac{\partial r}{\partial \psi}\right)^{2}\right]^{-3 / 2} \\
- & {\left[\left(\frac{\partial r}{\partial \phi}\right)^{2}+r^{2}\left(\frac{\partial r}{\partial \psi}\right)^{2}\right]^{-1 / 2}\left|\frac{\partial r}{\partial \psi}\right| }
\end{aligned}
$$

Exploiting the anticipated left/right symmetry of the waves, we choose to solve over half of the domain, $0 \leq \phi<\infty$, and impose the symmetry condition

$$
\frac{\partial r}{\partial \phi}=0
$$

at $\phi=0$.

The calculations were performed by first discretizing (6.6) using centred differences over a truncated rectangular grid, with $N$ equally-spaced mesh points in $\phi$ over the range $\left[0, \phi_{\infty}\right]$, where $\phi_{\infty}$ is a specified truncation level, and with $M$ equally-spaced mesh points in $\psi$ over the range $\left[0, \psi_{S}\right]$. The Bernoulli condition (6.8) is discretized using backward differences for the $\psi$-derivatives and centred differences for the $\phi$-derivatives (and using the symmetry condition (6.10) at the mesh points on $\phi=0$, and imposing the condition that $\partial r / \partial \phi=0$ at $\left.\phi=\phi_{\infty}\right)$. Enforcing the discretized form of (6.6) at the interior meshpoints, and the boundary conditions at the edges as described, we compile a system of $N M$ nonlinear algebraic equations which we solve using Newton's method. The free surface location is extracted from the converged solution as $S(\phi)=r\left(\phi, \psi_{S}\right)$. For some calculations, it is convenient to fix the amplitude of the wave by prescribing the value of $r$ at $\phi=0, \psi=\psi_{S}$ and treating $c$ as an additional unknown to be found as part of the solution. In this case we first rescale $\psi \rightarrow c \psi$ to ensure a fixed domain size, and then proceed as already described. Finally, the shape of the free surface is obtained in physical space by integrating the second of (6.4) at $\psi=\psi_{S}$ using the trapezium rule.

For the results presented in the next section we typically use $N=40$ and $M=20$, and $\phi_{\infty}=9$, but some adjustment to these values is needed for some of the calculations. For calculations where intricate details of the profile need to be accurately resolved, we 
(a)

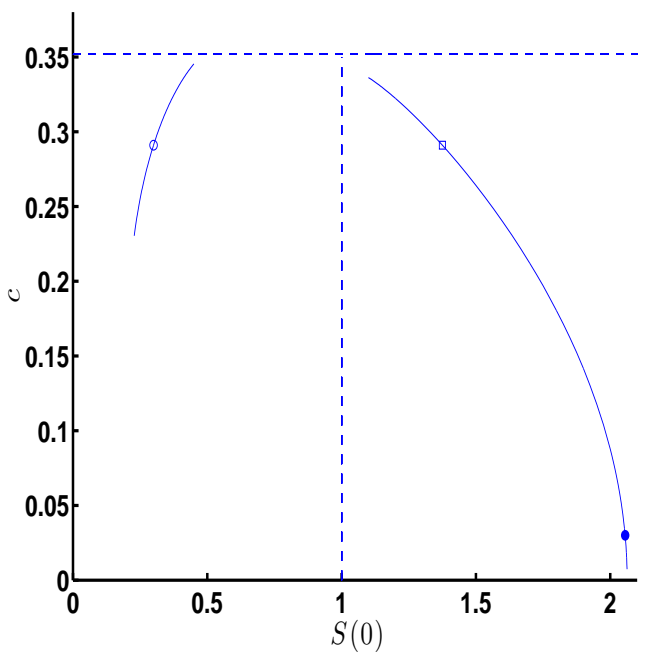

Solitary waves on a ferrofluid jet

(b)

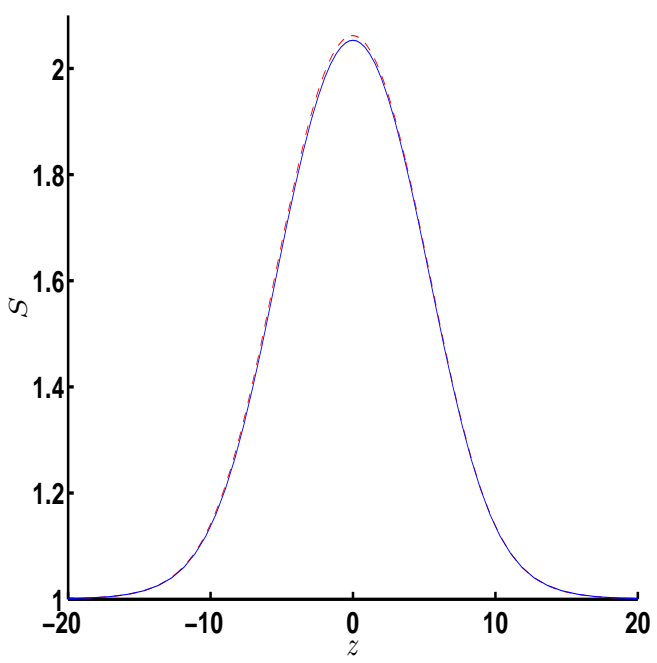

Figure 4. (a) Solution branches for $B=1.25$ and $b=0.1$; the vertical broken line indicates a uniform stream, and the horizontal broken line indicates $c_{0}=0.352$ according to (3.4). (b) Surface profile $S(z)$ for $B=1.25$ and $b=0.1$, with $c=0.03$, corresponding to the dot on the curve in $(a)$; the broken line is the corresponding static solution.

found it convenient to use a non-uniform grid in $\phi$ so as to be able to focus computational points where needed. In order to latch onto the various solitary wave solution branches, we found it necessary to employ a method used by a number of authors. An artificial Gaussian pressure distribution is included in the Bernoulli condition (2.6) on the free surface to force a solution different to a uniform stream. Removing the artificial pressure, this solution can then be used as the initial guess to lock onto the required solitary wave solution branch. Particular details of the method are described in, for example, VandenBroeck \& Dias (1992) and Maleewong et al. (2005).

\section{Nonlinear calculations}

We begin with a discussion for the case $B=1.25$ and $b=0.1$. According to the small-amplitude weakly nonlinear theory of RE, at this Bond number we expect to find elevation solitary waves (with $S(0)>1$ ) for $c<c_{0}=0.352$. This branch of solution should bifurcate from zero amplitude at $c=c_{0}$. In fact not only have we computed waves on this bifurcation branch, we have also identified a new branch of nonlinear solutions corresponding to depression waves (with $S(0)<1$ ). Figure $4(a)$ shows both of these solution branches for $B=1.25$. The rightmost branch, corresponding to the elevation waves, should bifurcate from the uniform stream at $c=c_{0}, S(0)=1$ according to RE's weakly-nonlinear theory. However, as the bifurcation point is approached, the wave profile becomes increasingly wide, demanding an increasingly large computational domain and, ultimately, it is impractical to follow the branch numerically all the way to the point $S(0)=1$. Following this solution branch as the amplitude $S(0)$ increases, the wave speed $c$ approaches zero. The wave profile at $c=0.03$ is shown in figure 4 $(b)$ and compared with the static solution at the same Bond number computed using the method discussed in section 4. The agreement between the two profiles is excellent, providing strong evidence that a limiting profile with zero wave speed and finite amplitude is eventually reached along this branch. 
(a)

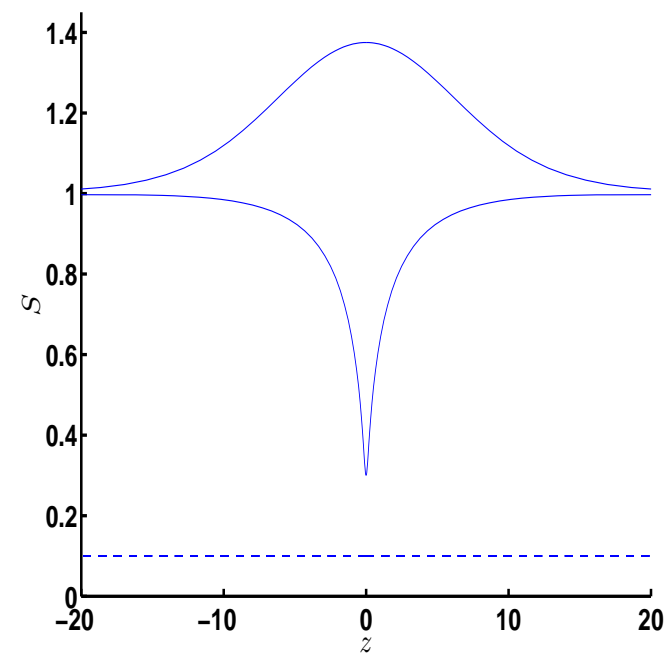

(b)

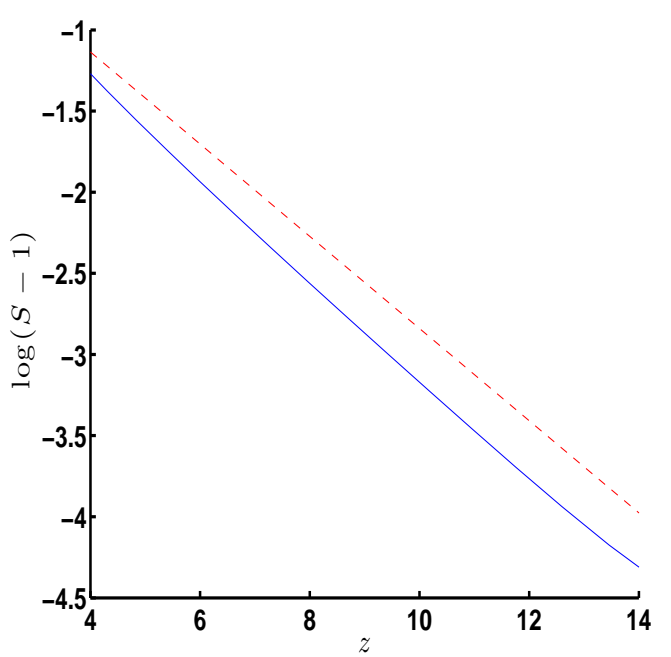

Figure 5. (a) Surface profile $S(z)$ for $B=1.25$ and $b=0.1$ corresponding to the circle (depression wave with $c=0.29$ ) and the square (elevation wave with $c=0.29$ ) on the curve in figure $4(a)$. The broken line shows the location of the axial rod. $(b) \log (S-1)$ against $z$ for the elevation wave in $(a)$ of the current figure. The broken line has slope -0.284 , corresponding to the far-field decay rate, and is shown for comparison purposes.

The new branch of nonlinear solutions, corresponding to the leftmost curve in figure 4( $a$ ), apparently bifurcates from finite amplitude at $c=c_{0}$ and at approximately $S(0)=$ 0.48. It is extremely difficult computationally to follow this branch back to the bifurcation point for the same reason stated in the previous paragraph. Indeed, it can be seen from figure $3(a)$ that the exponential far-field rate for the wave profile tends to vanish as $c \rightarrow c_{0}$, so that an impractically large computational domain is required in this limit. Following the nonlinear branch in the other direction toward smaller values of $S(0)$, we find that the wave profile eventually reaches a limiting configuration with a trapped bubble (see figure 6] for an example of this sort of profile). Reducing $S(0)$ further results in a self-intersecting wave profile with no meaningful physical interpretation. Sample wave profiles at the same wave speed on the elevation and depression branches are shown in figure 5 $(a)$. In figure 5 (b) the rate of decay of the elevation profile in the far-field is compared favourably with the theoretical decay rate computed as described in section 5 . The decay rate for the depression profile in figure 5( $a$ ) is the same as for the elevation profile.

When $b=0.1$, we have $B_{2} \approx 9$ and we expect that $B_{1} \approx 1.5$. Note that RE found $B_{1}=$ 1.5 for $b=0$; for general $b$, difficulties already noted above in computing solution branches close to the uniform stream hamper attempts to determine the general dependence of $B_{1}$ on $b$. In the range $1.5<B<9$ we expect to find small-amplitude depression waves. For $1.5<B<2$ we find both depression waves and elevation waves. The depression waves bifurcate from the uniform stream according to RE's weakly-nonlinear theory. The elevation waves correspond to a solution branch which bifurcates nonlinearly from finiteamplitude. So, effectively, the scenario here is the same as for the range $1<B<B_{1} \approx 1.5$ discussed above but with the roles of the two solution branches reversed. Figure 6 shows the limiting depression wave profile for $B=2$ and $b=0.1$ and, as the inset makes clear, there is a trapped toroidal bubble in the middle. The solution space for the case $B=1.75$ and $b=0.1$ is shown in figure 7 . The limiting depression wave profile has a trapped 


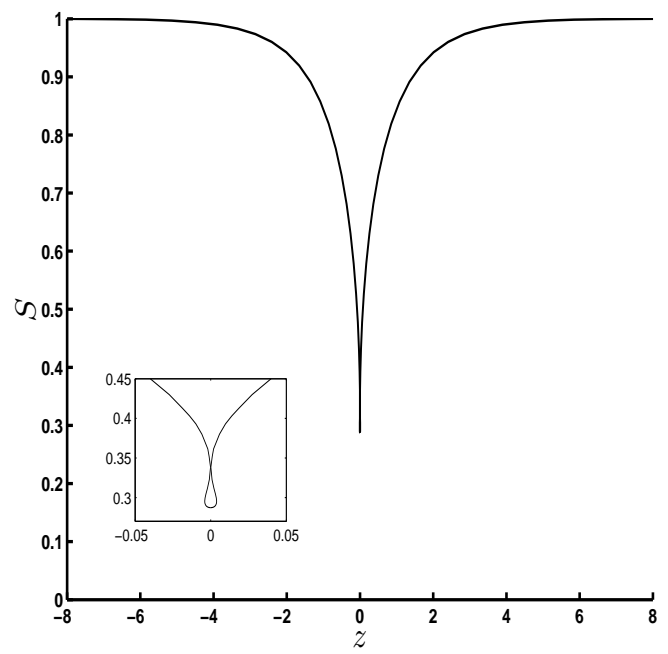

FiguRE 6. A depression wave profile with a trapped bubble for $B=2$ and $b=0.1$; the inset shows a close up of the bubble.

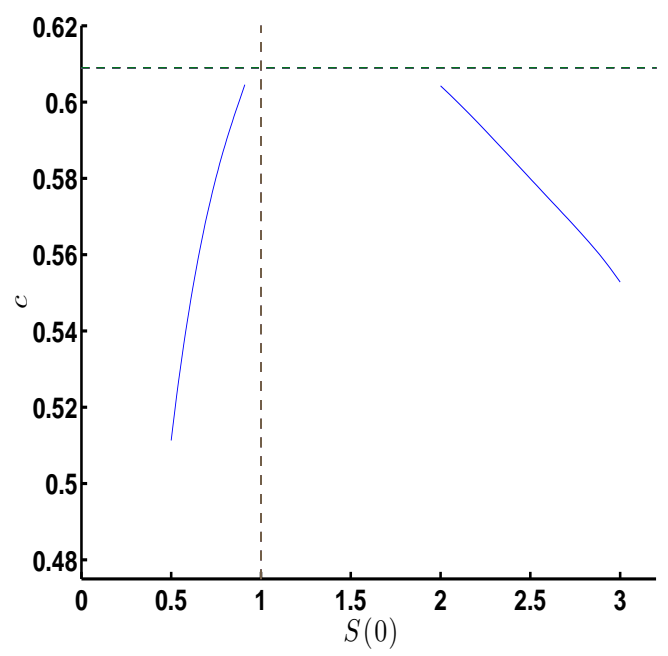

Figure 7 . Solution branches for $B=1.75$ and $b=0.1$; the vertical broken line indicates a uniform stream, and the horizontal broken line indicates $c_{0}=0.609$ according to (3.4).

bubble in the middle (similar to that seen in figure 6). Following the elevation wave branch, eventually a static profile is reached as $c \rightarrow 0$. At $B=2$, the nonlinear branch of elevation waves does not end in a static profile since no static profile exists at this Bond number according to section 4 instead it appears that the wave amplitude continues to increase (we computed waves of amplitude up to 6 in the current dimensionless units). In the range $2 \leq B \leq B_{2}$, we have only been able to find depression waves on a branch which bifurcates from the uniform stream. A sample profile is shown in figure 8 for the case $B=4$ and $b=0.1$.

For $B \geq B_{2}$ the decay rates computed in section 5 indicate that the waves travelling at a speed lower than $c_{M}$ do not return monotonically to the uniform level but have decaying oscillations in the far-field. Specifically, for $B=30$ and $b=0.1$, the decay rate is complex in the range $1.1<c<c_{M}=3.1$ (see figure $3(b)$ ). Non-monotonic decay is evident in the 


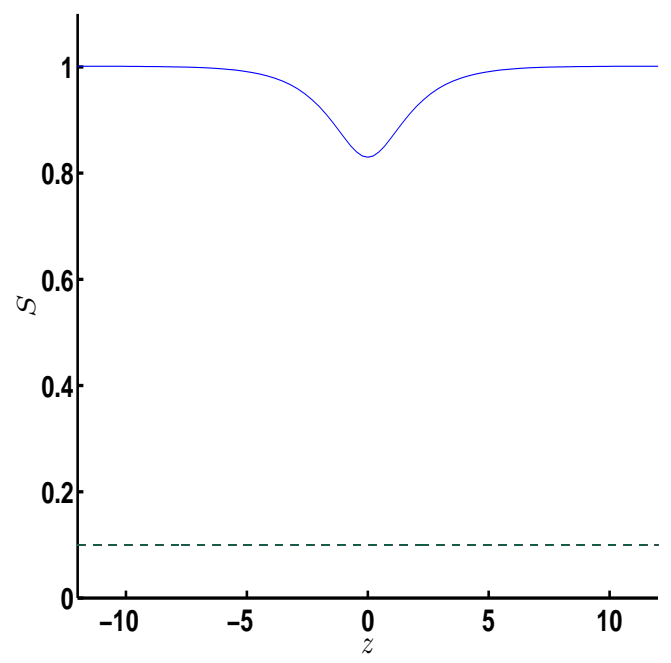

Figure 8. A depression wave profile for $B=4.0$ and $b=0.1$ with wave speed $c=1.15$.

(a)

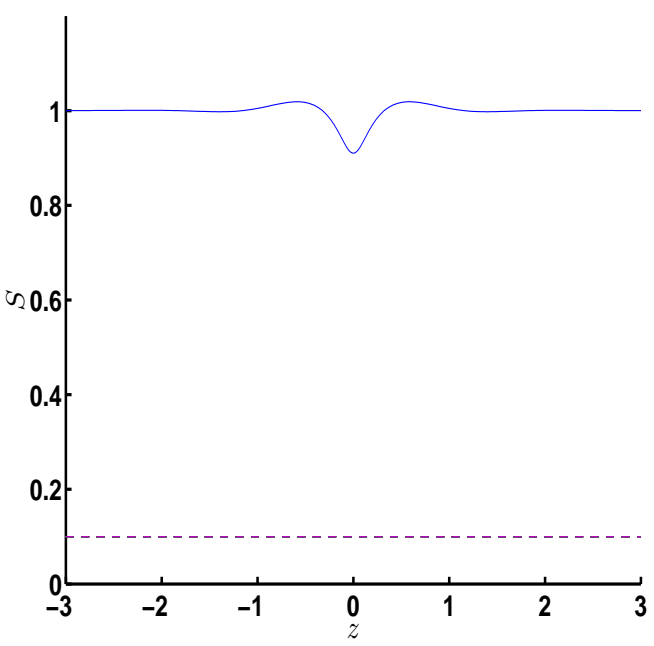

(b)

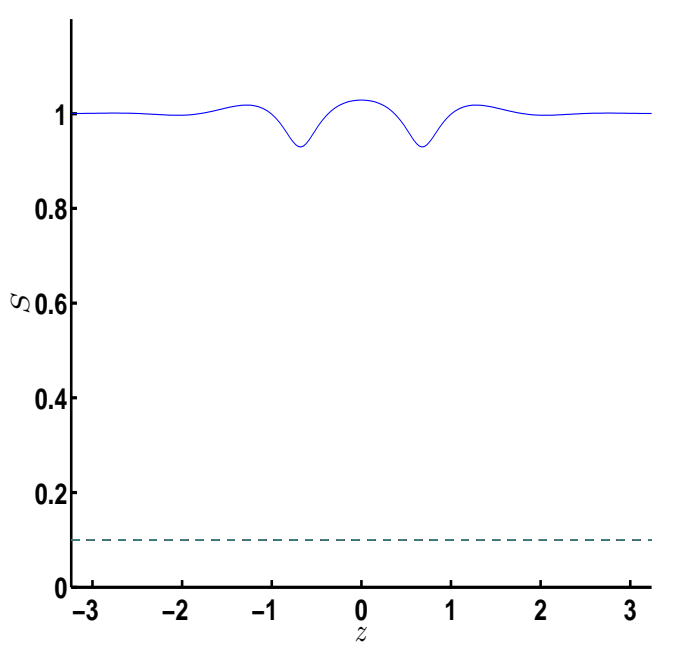

FiguRE $9 . \quad B=30$ and $b=0.1$ : (a) Depression wave profile with wave speed $c=2.92$ corresponding to the circle on the depression branch in figure 10. (b) Elevation wave profile with wave speed $c=2.98$ corresponding to the square on the depression branch in figure 10 .

depression-wave profile shown in figure $9(a)$ for $B=30$ and $b=0.1$. Elevation profiles (with $S(0)>1$ ) are also possible for $B \geq B_{2}$ and an example is shown in figure $9(b)$ for $B=30$ and $b=0.1$. The profiles in figure 9( $a)$ and $9(b)$ correspond to points on opposing arms of the same solution branch. This solution branch is shown in figure 10, Attempts to follow this branch into the point $S(0)=1$ (from either the left or the right) are frustrated by the computational difficulties discussed above. However, there is very good reason to suppose that the two solid curves in this figure meet smoothly at the point $S(0)=1$, $c=c_{M}$, where the vertical and horizontal broken lines cross. Our reasoning comes by drawing an analogy with two-dimensional gravity-capillary waves. In this case a nonlinear Schrödinger equation describes the weakly-nonlinear dynamics around the minimum on the dispersion curve (see the discussion in section 3) and this equation predicts that both 


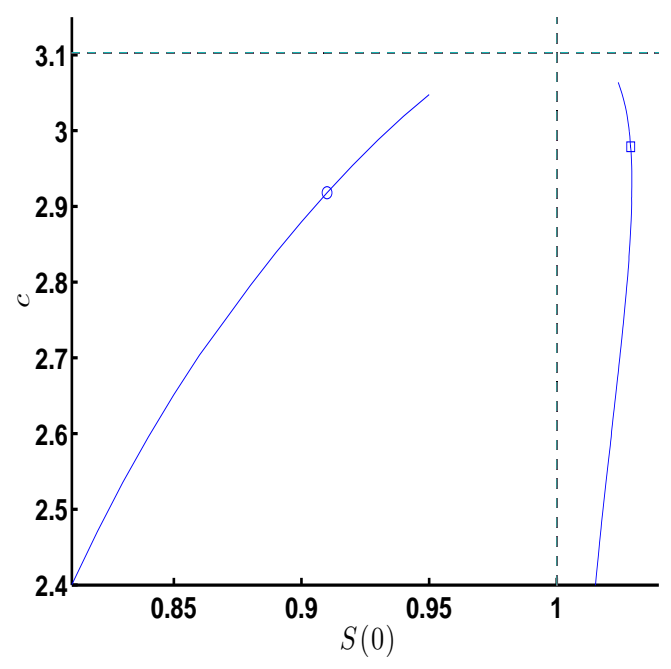

FiguRE 10. Solution branch for $B=30$ and $b=0.1$; the vertical broken line indicates a uniform stream, and the horizontal broken line indicates $c_{M}=3.10$ according to (3.4). The wave profile corresponding to the circle/square symbol is shown in figure $9(a) 9(b)$ respectively.

an elevation and a depression branch should bifurcate from the uniform stream at this minimum point. An analogous equation for the present problem is not available, but we suspect that the same will be true here.

Moving in the opposite direction along the branches in figure 10, we find that on the left-hand branch a limiting profile is finally attained and it has a trapped bubble, as in figure 6. Giving an accurate description of what happens along the right-hand branch is more delicate. Our most accurate computations predict that this branch also ends in a wave profile with a trapped bubble. However, experience from two-dimensional gravitycapillary waves serves as a cautionary warning. For such waves Vanden-Broeck \& Dias (1992) predicted that a similar solution branch ends in a limiting profile with a trapped bubble, but more accurate calculations performed by Dias et al. (1996) showed that in fact the branch continues and executes many loops and turns before it becomes computationally impractical to continue further. Wang et al. (2013) found similar behaviour for two-dimensional flexural-gravity waves.

The decay rate graph in figure $3(b)$ indicates that when $c<1.1$ the decay rate is real and monotonic decay in the far-field is expected. We investigated the possibility that further solitary waves solutions exist in this range with monotonically decaying tails. In this case, following the solution branch down to the relevant range of $c$ proved extremely difficult. However, as $c$ is lowered from $c_{M}=3.1$ the wave profiles eventually self-intersect at some value in the range $1.1<c<3.1$ and although we were not able to obtain accurate profiles, it seems likely that profiles in the range $0<c<1.1$ also self-intersect.

In section 4 we noted that when $B>2$ there are no smooth static profiles, but static profiles which meet the axial rod in a cusp are possible. We investigated whether such a static profile may be approached dynamically as the wave speed tends to zero. Figure 11 shows the limiting profile which is reached for the case $B=2.5$ and $b=0.45$. The broken curve in the figure represents the static profile which corresponds to the heteroclinic orbit connecting two neighbouring pairs of equilibrium points in the static phase space. In figure 2 $(b)$ it was shown how part of this profile may be interpreted as a wave profile with a cusp. The convincing overlap of the two curves in figure 11 is strong evidence that a cusped static profile is indeed approached in the limit as the wave amplitude $S(0)$ 


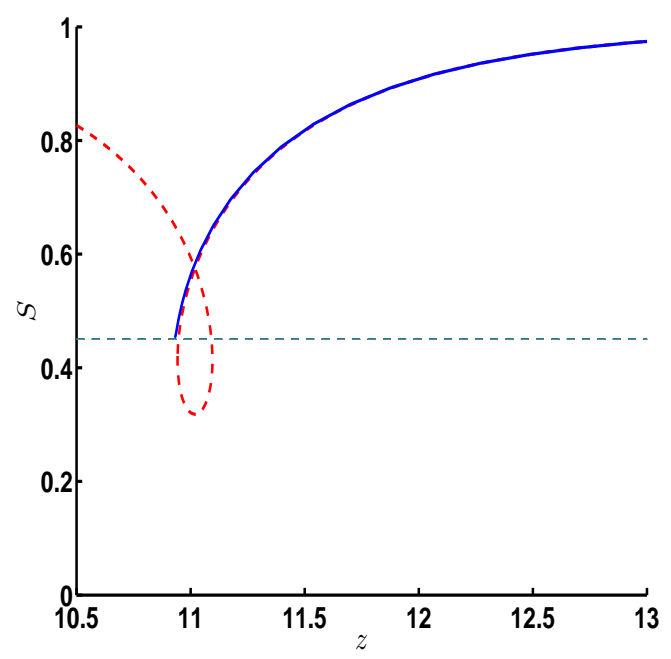

FiguRE 11. Near-limiting profile (solid curve) for $B=2.5$ and $b=0.45$. The shown profile has $S(0)=0.453$ and wave speed $c=0.07$. The broken curve is the static solution (see section 4). The horizontal broken line shows the location of the axial rod.

approaches 0.45 from above and the wave speed approaches zero. Note that although the profiles agree over most of the range, near to the point of contact with the wall the two solutions necessarily deviate from one another because of the symmetry condition (6.10) imposed in the dynamic calculation, which prevents the profile from approaching a true cusp.

\section{Discussion}

We have investigated the propagation of axisymmetric solitary waves on a ferrofluid jet in the presence of a magnetic field generated by a current-carrying metallic rod mounted along the jet axis. In particular, we have used a numerical method to solve the full problem and to extend the weakly-nonlinear results of RE into the fully-nonlinear regime. We have confirmed that, depending on the size of the magnetic Bond number, elevation and depression solitary waves exist and appear as bifurcations from the cylindrical uniform stream solution. Moreover, we have identified a number of new solution branches which do not bifurcate from the uniform stream but appear at finite amplitude. Such branches have previously been identified for two-dimensional interfacial gravity-capillary waves by Laget \& Dias (1997). A summary of the wave characteristics for different values of the Bond number is presented in Table 1. We have also demonstrated that along a given solution branch a limiting profile may be reached with either a trapped toroidal bubble, or with a cusp if the wave makes contact with the solid rod. Alternatively the limiting profile may correspond to a smooth solution of the static problem.

Bourdin et al. (2010), henceforth referred to as BBF, reported experimental observations of axisymmetric solitary waves on a ferrofluid. The experimental set-up is essentially identical to the theoretical scenario studied by RE and also studied in the present work. One important difference is that, in order to mitigate against the effect of gravity, in the experiments the ferrofluid jet was surrounded by an immiscible outer fluid of almost the same density. In the present notation, BBF's experimental parameters correspond to $b^{*}=1.5 \mathrm{~mm}$ and $a^{*}=3.3 \mathrm{~mm}$ (for their solitary wave experiments) or $a^{*}=3.8 \mathrm{~mm}$ (for their linear wave experiments), so that $b=0.45$ (solitary waves) and $b=0.39$ (linear 
Wave type

$\begin{array}{cc}1<B<B_{1} & \text { Elevation waves / Depression waves (nonlinear) } \\ B_{1}<B \leq 2 & \text { Elevation waves (nonlinear) / Depression waves } \\ 2<B<B_{2} & \text { Depression waves } \\ B>B_{2} & \text { Elevation waves/Depression waves }\end{array}$

TABLE 1. Summary of the wave types found across various ranges of the Bond number $B$. Depression waves have $S(0)<1$ and elevation waves have $S(0)>1$. The qualifier 'nonlinear' means that these solutions bifurcate from finite-amplitude and not from the uniform stream. The threshold value $B_{2}$ (and presumably $B_{1}$ ) depends on the rod radius $b$. For $b \rightarrow 0, B_{1}=1.5$ and $B_{2}=9$ (Rannacher \& Engel 2006).

waves). In either case $b$ is not particularly small. This is important since BBF relate their observations to theoretical results based on the limit $b \rightarrow 0$. In their figure 3 , they report experimentally-determined values of the linear wave speed $c_{0}$ for small-amplitude waves over a range of Bond numbers. With one exception, all of their data points for $c_{0}$ lie below the expected theoretical prediction based on taking $b=0$. We suggest that an explanation for this is provided by formula (3.4), which shows that the actual linear wave speed is lower than this by a factor $\left(1-b^{2}\right)^{1 / 2}=0.92$ for $b=0.39$. On making this correction, we find that the theoretical prediction in their figure 2 passes very convincingly through the experimental data points.

Considering that BBF's experiments were not performed in a vacuum, but with a second, outer fluid, we are motivated to see if this makes a significant difference to the theoretical predictions. Repeating the linearised analysis in section 3 for the case when the ferrofluid jet is surrounded by a second inviscid fluid of equal density and of infinite extent with zero magnetic susceptibility $(\chi=0)$, so that it feels no magnetic body force, we derive the following dispersion relation to replace (3.3),

$$
c^{2}=\frac{1}{k}\left(\frac{m_{1} K_{1}(k)}{m_{1} K_{0}(k)+m_{2} K_{1}(k)}\right)\left(k^{2}-1+B\right),
$$

(see Arkhipenko et al. 1980) where $m_{1}=I_{1}(k) K_{1}(k b)-I_{1}(k b) K_{1}(k)$ and $m_{2}=I_{1}(k b) K_{0}(k)+$ $I_{0}(k) K_{1}(k b)$. Carrying out a small- $k$ expansion of (8.1), we find that

$$
c^{2}=\frac{1}{2}\left(1-b^{2}\right)(B-1)+\frac{1}{4}\left(1-b^{2}\right)^{2}(B-1) k^{2} \log k+O\left(k^{2}\right) .
$$

From this we can see that the slope $\mathrm{d} c / \mathrm{d} k<0$ if $B>1$. Since $c^{2} \rightarrow \infty$ as $k \rightarrow \infty$, we deduce that the $c-k$ dispersion curve has a minimum if $B>1$, that is over the whole range of interest in the Bond number. We recall that for a jet in a vacuum, a minimum in the dispersion curve appears only when $B>B_{2}(b)$. Figure 12 shows a comparison of dispersion curves for a jet in a vacuum (with $b=0$ and with $b \neq 0$ ) according to (3.3), and for a jet in a surrounding fluid according to (8.1). Figure 12( $a)$ is plotted at $B=10.5$, which is one of the values looked at by BBF. The uppermost curve meets the vertical axis at a different point to the two lower curves because of the different values of $c_{0}$ according to (3.4). Notice that the single-fluid curve for $b=0$ has a minimum but the single-fluid curve for $b=0.45$ does not have a minimum (the minimum appears at 
(a)

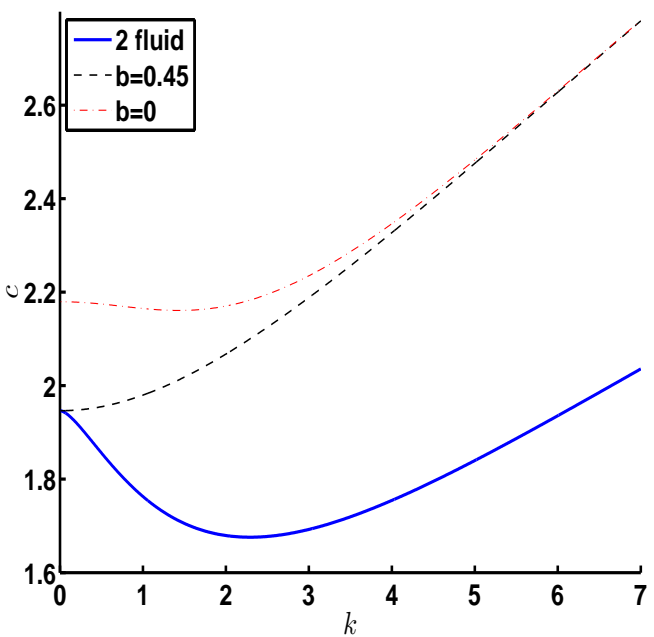

(b)

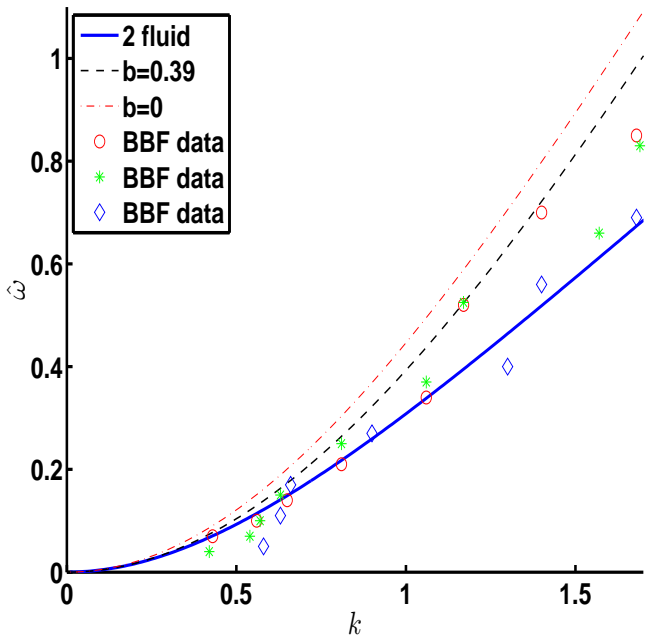

Figure 12. Comparison of dispersion curves for the single-fluid and two-fluid models: $(a) c$ versus $k$ for $B=10.5$ and for a single fluid (with $b=0$ and $b=0.45$ ) and for two fluids with $b=0.45$. (b) $\hat{\omega}=\omega^{2} /\left(k^{2}-1+B\right)$ versus $k$, where $\omega=k c$, for the single-fluid model with $b=0$ and $b=0.39$ and for the two-fluid model with $b=0.39$. The symbols in $(b)$ correspond to experimental data points taken from figure 2 of BBF (we have used the same symbols as them).

higher Bond number). The axes in figure $12(b)$ have the same ordinate as in figure 2 of $\mathrm{BBF}$, which has been scaled to remove the effect of the Bond number. Also included in this figure are some of BBF's experimental data points which we have taken from their figure 2. It does appear that the solid curve for the two-fluid model provides the more convincing fit with the experimental results. We may conclude that the effect of the outer fluid in the experiments is significant and should not be overlooked when drawing a comparison with theory.

BBF carried out solitary wave experiments for $B=8.3$ and $B=10.5$, with apparatus for which $b=0.45$ (see above). It appears that these Bond numbers were chosen to lie on either side of the critical value $B=9$, which was identified by $\mathrm{RE}$ as the point at which the wave characteristics are expected to change (see section 5 of $\mathrm{RE}$ and Table 1 of BBF). We find that the appropriate critical value is in fact $B_{2}(0.45)=15.4$, so that the Bond numbers used in BBF's solitary wave experiments actually lie in the range $2 \leq B \leq B_{2}$. What is particularly interesting is that $\mathrm{BBF}$ report finding depression waves for $B=8.3$ and elevation waves for $B=10.5$. The latter is puzzling as it does not accord with our predictions: we expect only $\mathrm{KdV}$-type depression waves in the range $2 \leq B \leq B_{2}$ (see table 1). However, given the remarks made in the previous paragraph, it seems plausible that the discrepancy between BBF's experimental solitary wave observations and our nonlinear results can be explained by the fact that the outer fluid is absent in our calculations. We are led to conjecture that the elevation/depression waves seen in BBF's experiments correspond to solitary waves on the solution branch which bifurcates from the minimum of the dispersion curve (for the two-fluid model, this is the lowermost curve in figure 12 $a$ ). Such waves travel at a speed $c<c_{M}$, where $c_{M}$ is the minimum value on this curve. An alternative explanation is that BBF observed generalised solitary waves with non-decaying oscillations in the far-field; such waves travel with speed $c>c_{0}$ (see, for example, Beale (1991), Vanden-Broeck (1991) and Hunter \& Vanden-Broeck (1983) for discussions of generalised solitary waves in two-dimensional flow). It would 
be very interesting to extend the present computations to allow for generalised solitary wave solutions, and to include the effect of an outer fluid of equal density. These are the subject of current investigation.

\section{REFERENCES}

Akylas, T. R. 1993 Envelope solitons with stationary crests. Phys. Fluids 5, 789-791.

Arkhipenko, V. I. \& Barkov, Yu. D. 1980 Experimental study of the breakdown of the cylindrical layer of a magnetizable fluid under the action of magnetic forces. J. Appl. Mech. Tech. Phys 21, 98-105.

Arkhipenko, V. I., Barkov, Yu. D., Bashtovoi, V. G. \& Krakov, M. S. 1980 Investigation into the stability of a stationary cylindrical column of magnetizable liquid. Fluid Dynamics 15, 477-481.

Bashtovoi, V. G. \& Foigel, R. 1983 Solitary and cnoidal waves in ferrofluid. Magnetohydrodynamics 19, 160-165.

Bashtovoi, V., Rex, A. \& Foigel, R. 1983 Some non-linear wave processes in a magnetic fluid. J. Magn. Magn. Mater. 39, 115-118.

Beale, T. J. 1991 Solitary water waves with capillary ripples at infinity. Commun. Pure Appl. Maths, 64, 211257.

Bourdin, E., BACRI, J.-C. \& FAlCON, E. 2010 Observation of axisymmetric solitary waves on the surface of a ferrofluid. Phys. Rev. Lett. 104, 094502.

Dias, F. \& Kharif, C. 1999 Nonlinear gravity and capillary-gravity waves. Ann. Rev. Fluid Mech. 31, 301-346.

LAGET, O. \& DiAs, F. 1997 Numerical computation of capillary-gravity interfacial solitary waves. J. Fluid Mech., 349, 221-251.

Dias, F., Menace, D. \& Vanden-Broeck, J.-M. 1996 Numerical study of capillary-gravity solitary waves. Eur. J. Mech. 15, 17-36.

JEPpson, R. W. 1970 Inverse formulation and finite difference solution for flow from a circular orifice J. Fluid Mech. 40, 215-223.

Hunter, J. K. \& Vanden-Broeck, J.-M. 1983 Solitary and periodic gravity-capillary waves of finite amplitude. J. Fluid Mech. 134, 205-219.

Maleewong, M., Asavanant, J. \& Grimshaw, R. 2005 Free surface flow under gravity and surface tension due to an applied pressure distribution: I Bond number greater than onethird. Theor. Comp. Fluid Dyn. 19, 237-252.

Miles, J. W. 1980 Solitary waves. Ann. Rev. Fluid Mech. 12, 11-43.

Osborne, T. \& Forbes, L. K. 2001 Large amplitude axisymmetric capillary waves. In IUTAM Symposium on Free Surface Flows, 221-228.

PĂRĂU, E. I., VANDEN-BroeCK, J.-M. \& CoOKer, M. J. 2005 Nonlinear three-dimensional gravity-capillary solitary waves. J. Fluid Mech. 536, 99-105.

Rannacher, D. \& Engel, A. 2006 Cylindrical Korteweg-de Vries solitons on a ferrofluid surface. New J. Phys. 8, 108-123.

RADWAm, A. E. 1988, Hydrodynamic stability of an annular liquid jet having a mantle solid axis using the energy principle. Indian J. Pure Appl. Math. 19, 1105-1114.

Rayleigh, J. W. S. 1878 On the instability of jets. Proc. Lond. Math. Soc. 10, 4-13.

Rosensweig, R. E. 1985, Ferrohydrodynamics, Dover, New York.

Strogatz, S. H. 2000, Nonlinear Dynamics and Chaos, Perseus Books, Jackson.

VAnden-Broeck, J.-M 1991 Elevation solitary waves with surface tension. Phys. Fluids A 3(11), 2659-2663.

VAnden-Broeck, J.-M. 2010, Gravity-capillary free-surface flows, Cambridge University Press, Cambridge.

Vanden-Broeck, J.-M., Miloh, T. \& Spivack, B. 1998 Axisymmetric capillary waves. Wave Motion 27, 245-256.

VANDEN-Broeck, J.-M. \& Dias, F. 1992 Gravity-capillary solitary waves in water of infinite depth and related free-surface flows. J. Fluid Mech. 240, 549-557.

Wang, Z., Vanden-Broeck, J.-M. \& Milewski, P. 2013 Two-dimensional flexural-gravity waves of finite amplitude in deep water. IMA J. Appl. Math. 78, 750-761. 